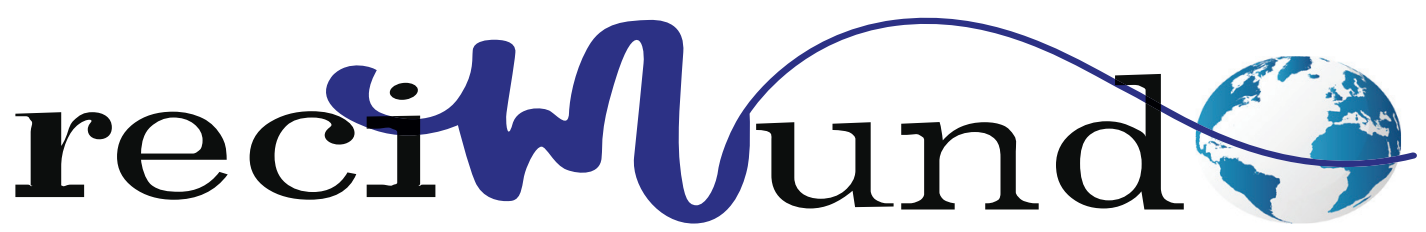

Revista Científica Mundo de la Investigación y el Conocimiento

DOI: 10.26820/recimundo/4.(1).enero.2020.249-266

URL: http://recimundo.com/index.php/es/article/view/726

EDITORIAL: Saberes del Conocimiento

REVISTA: RECIMUNDO

ISSN: 2588-073X

TIPO DE INVESTIGACIÓN: Artículo de Revisión

CÓDIGO UNESCO: 32 Ciencias Médicas

PAGINAS: 249-266

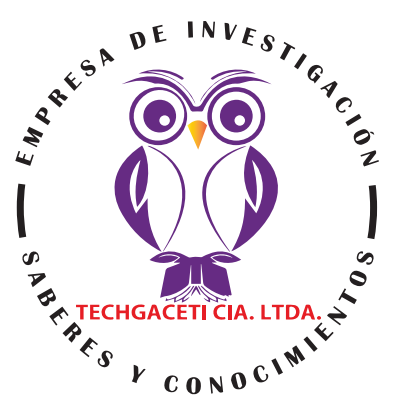

\title{
Manual de prácticas de farmacología
}

\author{
Manual of pharmacology practices \\ Manual de práticas farmacológicas
}

Oscar Fernando Lituma Peñarreta ${ }^{1}$; Elsy Ivonne Avalos Moreno²; Alex Humberto Zea Morales; Raúl Germán Castro García ${ }^{4}$

RECIBIDO: 18/09/2019 ACEPTADO: 29/10/2019 PUBLICADO: 31/01/2020

1. Magister en Gerencia Educativa; Doctor en Medicina y Cirugia; Universidad de Guayaquil; Guayaquil, Ecuador; oscar. litumap@ug.edu.ec; iD https://orcid.org/0000-0003-3194-1543

2. Especialista en Medicina Interna; Especialista en Gerencia y Planificación Estratégica de Salud; Doctora en Medicina y Cirugia; Universidad de Guayaquil; Guayaquil, Ecuador; elsy.avalosm@ug.edu.ec; iD https://orcid.org/0000-0002-5242-3764

3. Médico; Investigador Independiente; Guayaquil, Ecuador; alexzea_md@hotmail.com; iD https://orcid.org/0000-00031876-1548

4. Magister en Diseño Curricular; Licenciado en Terapia Respiratoria; Tecnólogo Médico en Terapia Respiratoria; Universidad de Guayaquil; Guayaquil, Ecuador; raul.castrog@ug.edu.ec; (D) https://orcid.org/0000-0003-4315-2031

CORRESPONDENCIA

Oscar Fernando Lituma Peñarreta

oscar.litumap@ug.edu.ec

Guayaquil, Ecuador 


\title{
RESUMEN
}

Una actualización de los ensayos de investigación experimentales farmacológicas es menester para cualquier instituto de enseñanza y de trabajo experimental de farmacología. Es idóneo pensar que los experimento clínico y todo lo que implica, debe contar con los recursos y el conocimiento apropiado desde la fase de ensayo inicial. Así mismo, la formación de profesionales que cuenten con tan importante conocimiento y práctica farmacológica, es de extrema importancia para la salud humana. El presente artículo expone el Manual de Prácticas Experimentales de Farmacología de la Facultad de Ciencias Médicas de la Universidad de Guayaquil como una propuesta alternativa en pos del desarrollo del profesional médico de la UG, y para romper con moldes obsoletos de enseñanzas que obstruyen el desarrollo investigativo de la farmacología en la Universidad.

Palabras clave: Manual, Farmacología, Ensayo, Investigación, Experimentos.

\begin{abstract}
An update of the experimental pharmacological research trials is necessary for any institute of teaching and experimental work of pharmacology. It is ideal to think that the clinical experiment and all that it implies, must have the resources and the appropriate knowledge from the initial trial phase. Likewise, the training of professionals who have such important knowledge and pharmacological practice is extremely important for human health. The present article exposes the Manual of Experimental Practices of Pharmacology of the Faculty of Medical Sciences of the University of Guayaquil as an alternative proposal for the development of the medical professional of the UG, and to break with obsolete molds of teaching that obstruct the research development of pharmacology at the University.
\end{abstract}

Keywords: Manual, Pharmacology, Essay, Research, Experiments.

\section{RESUMO}

Uma atualização dos ensaios de pesquisa farmacológica experimental é necessária para qualquer instituto de ensino e trabalho experimental de farmacologia. É ideal pensar que o experimento clínico e tudo o que ele implica devem ter os recursos e o conhecimento apropriado da fase inicial do teste. Da mesma forma, a formação de profissionais com conhecimento e prática farmacológica tão importantes é de extrema importância para a saúde humana. Este artigo apresenta o Manual de Práticas Experimentais de Farmacologia da Faculdade de Ciências Médicas da Universidade de Guayaquil como uma proposta alternativa para o desenvolvimento do profissional médico da UG e romper com moldes obsoletos de ensino que dificultam o desenvolvimento da pesquisa. de farmacologia na Universidade.

Palavras-chave: Manual, Farmacologia, Ensaio, Pesquisa, Experimentos. 


\section{Introducción}

Es la industria farmacológica una de las más poderosas e influyentes en la vida humana, y el avance tecnológico la ha llevado de la mano porque siempre ha estado en constante desarrollo. Esta imparable área de la investigación científica, tiene sus cimientos en innumerables recursos humanos, profesionales que se han comprometido con la investigación, básicamente, experimental.

La formación de profesionales en el área de farmacología, incluyendo médicos, enfermeras y demás profesionales de la salud, debería ser un asunto serio y en el que no debería escatimarse en gastos tanto materiales como intelectuales. Sin embargo, no todas las instituciones cuentan con el máximo aporte o recurso disponible para llevar a cabo su labor de manera óptima.

Las instituciones públicas que se dedican al estudio farmacológico están siempre orientadas a la salud pública y a la alimentación, cuentan con apoyo financiero suficiente según la capacidad de cada país. Los países desarrollados tienen mucho avance farmacológico mientras que los países más pobres o en vía de desarrollo cuentan con nada o casi nada para financiar sus estudios experimentales.

Por otro lado, las instituciones privadas son movidas por intereses lucrativos y su capital les permite la avanzada en estudios farmacológicos. Ni hablar de las grandes trasnacionales farmacéuticas que imperan en recursos de todo tipo para el desarrollo constante del área.

Todas las universidades del mundo que tienen una facultad de medicina o de farmacología, tienen seguro un departamento dedicado a la investigación y práctica farmacológica. No todas las universidades cuentan con el máximo de recurso posible y de vanguardia para desarrollar los estudios de farmacia, pero por lo menos deben contar con el incentivo investigativo para promocionar el experimento del área en cuestión.

En base a esto, los profesores, estudiantes y personal de laboratorio de la Facultad de Ciencias Médicas de la Universidad de Guayaquil, proponen un Manual de Prácticas Experimentales que incentiven la investigación farmacológica y promueva la mejora de las marchas y procedimientos experimentales de la cátedra.

En tal sentido, el objetivo que se persigue consiste en reprogramar el Manual de Prácticas Experimentales de Farmacología y promover una nueva cultura en el trabajo de investigación farmacológica. Serán necesarios experimentos más útiles que contemplen las drogas actuales, y para ello, se deberá disponer de los instrumentos y equipos indispensables para llevar a cabo los experimentos, así como la disposición de reactivos y materiales incluyendo al material biológico (animal).

El Manual de Prácticas Experimentales de Farmacología permitirá que el estudiantado sepa diferenciar el efecto terapéutico de los fármacos y medicamentos y evalúe el efecto biológico producido sobre aparatos y sistemas, mediante el método científico y siguiendo los procedimientos adecuados de laboratorio. Es decir, el futuro profesional de la Facultad de Ciencias Médicas de la Universidad de Guayaquil, deberá estar preparado para manejar los principales parámetros de la farmacología:

a) Farmacocinética: Es "el paso de las drogas a través del organismo en función del tiempo y de la dosis. Comprende los procesos de absorción, distribución, metabolismo o biotransformación y excreción de las drogas." (Malgor \& Valsecia, 2000, pág. 13)

b) Farmacodinámica: "es el estudio de los efectos bioquímicos y fisiológicos de los fármacos y sus mecanismos de acción. Los efectos de casi todos los medicamentos son consecuencia de su interacción con componentes macromoleculares del organismo son las acciones farmacológicas y los efec-

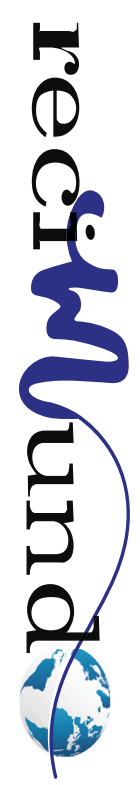


tos que producen los medicamentos en los organismos humanos sanos y enfermos." (Hilal-Dandan, Randa ; Brunton, Laurence, 2013, pág. 29).

c) Mecanismo de Acción: "Es el conjunto de procesos que se producen en las células, debido a las acciones de las drogas sobre el organismo. Las drogas tienen dos efectos fundamentales sobre el organismo, estimular o inhibir un proceso fisiológico. Los fármacos no producen nuevas funciones que el organismo no posee." (Chéry, 2010).

d) Efectos colaterales y secundarios que son los efectos distintos en el organismo a dosis normales.

e) Intoxicación o efectos indebidos de los fármacos en el organismo humano por dosis elevadas o tóxicas (sobredosificación).

f) Contraindicaciones: son situaciones normales o patológicas en las que no se puede administrar un medicamento.

g) Interacciones medicamentosas: al interactuar dos medicamentos pueden producir sinergismo (aumento de la acción farmacológica por la unión de dos drogas o antagonismo (disminución de la acción farmacológica por la adición de otra droga).

h) Preparados: Es la elección de la presentación comercial de medicamentos más adecuada.

i) Vías de administración: es la forma más óptima para administrar la droga al paciente, según el caso.

j) Dosificación: es la cantidad exacta necesaria de un fármaco que se debe administrar a un organismo sano o enfermo.

k) Indicaciones terapéuticas: Es la forma como debe aplicarse un determinado fármaco ante una enfermedad, además contempla la población a quién es dirigida. (niño, adulto, anciano).

\section{Metodología}

La propuesta presentó un enfoque cualitativo-cuantitativo (mixto). Desde el punto de vista cualitativo la desmotivación fue un factor preponderante. Se evaluó la calidad académica de la cátedra de farmacología de la Facultad de Ciencias Médicas de la Universidad de Guayaquil, comparando con el nivel de preparación y experimentación de otras instituciones en el mundo. De esta manera se considera obsoleta la actual estructura física, e insuficiente la cantidad instrumental, material, de reactivos y bioteros.

Se realizaron encuestas y cuestionarios de opinión sobre "las fallas pedagógicas en la docencia experimental de farmacología", dónde la población a estudiar fueron los docentes, estudiantes y personal de laboratorio de la Facultad de Ciencias Médicas de la Universidad de Guayaquil. Acompañando la modalidad cuantitativa se aplicó el método inductivo para analizar las características de la población para llegar a conclusiones generales.

Se realizó una exhaustiva revisión documental para el diseño del Manual de Prácticas Experimentales de Farmacología, la cual se tomó como base para fundamentar las Marchas Experimentales de Farmacología.

\section{Resultados}

Luego de la realización de encuesta y su cuestionario respectivo sobre las fallas pedagógicas en la docencia experimental de farmacología, aplicado sobre profesores, estudiantes y personal de laboratorio totalmente involucrados en el proceso enseñanza aprendizaje, se obtuvieron los siguientes resultados valorativos y cuantitativos:

- La mayoría considera inadecuadas las clases magistrales: poco tiempo de 4 horas semanales.

- Se debe hacer un reajuste en el pensum para la cátedra de farmacología. 
- Se habla de una pobre comunicación entre Estudiante y Profesor. Lo cual es preocupante dada la importancia de ello en el proceso de enseñanza y aprendizaje.

- Se necesita un reajuste en la evaluación teórico-práctica.

- Se considera obsoleta la estructura física del laboratorio.

- Escaso recursos materiales e Instrumentación.

- Insuficiente material biológico (ratones). Es necesario la construcción de un biotero con buena estructura y productividad.
- La mayoría coinciden en que las prácticas experimentales y fármacos usados deben actualizarse con tópicos útiles en la vida diaria del médico de la comunidad, quien tiene que resolver los problemas de la salud y provisión de medicamentos.

A partir de estos resultados es que surge la necesidad de diseñar un Manual de Prácticas de Farmacología Experimental, para llenar las aspiraciones de estudiantes, docentes y personal de apoyo de laboratorio, y como propuesta pedagógica, útil, actualizada con medicamentos cuyos efectos colaterales o secundarios sean minimizados en lo posible:

Tabla 1. Manual de prácticas experimentales de farmacología de la Facultad de Ciencias Médicas de la Universidad de Guayaquil

\begin{tabular}{|c|c|c|}
\hline \multicolumn{3}{|c|}{ TEMA } \\
\hline \multicolumn{2}{|r|}{ MARCHA } & TÍTULO \\
\hline 1 & $\begin{array}{l}\text { EXPERIMENTAL DE FARMACOLOGIA Y } \\
\text { ESTADISTICA }\end{array}$ & Cálculo dosis y diluciones \\
\hline 2 & $\begin{array}{l}\text { EXPERIMENTAL SISTEMA NERVIOSO } \\
\text { PERIFERICO }\end{array}$ & Anestésicos Locales \\
\hline 3 & $\begin{array}{l}\text { EXPERIMENTAL DEL SISTEMA } \\
\text { NERVIOSO CENTRAL }\end{array}$ & Anestésicos Generales. \\
\hline 4 & $\begin{array}{l}\text { EXPERIMENTAL DEL SISTEMA } \\
\text { NERVIOSO CENTRAL }\end{array}$ & Anticonvulsivantes \\
\hline 5 & $\begin{array}{l}\text { EXPERIMENTAL SISTEMA NERVIOSO } \\
\text { CENTRAL }\end{array}$ & Neurolépticos \\
\hline 6 & EXPERIMENTAL SISTEMA RENAL & Diuréticos \\
\hline 7 & $\begin{array}{l}\text { EXPERIMENTAL SISTEMA } \\
\text { CARDIOVASCULAR }\end{array}$ & Cardiotónicos \\
\hline 8 & $\begin{array}{l}\text { EXPERIMENTAL APARATO } \\
\text { DIGESTIVO }\end{array}$ & Antihelmínticos \\
\hline 9 & $\begin{array}{l}\text { EXPERIMENTAL SISTEMA } \\
\text { ENDOCRINO }\end{array}$ & Ocitócicos y útero inhibidores \\
\hline 10 & $\begin{array}{l}\text { EXPERIMENTAL SISTEMA } \\
\text { RESPIRATORIO }\end{array}$ & Histamina y antihistamínicos \\
\hline 11 & $\begin{array}{l}\text { EXPERIMENTAL APARATO } \\
\text { DIGESTIVO }\end{array}$ & $\begin{array}{l}\text { Sinergismo y antagonismo } \\
\text { medicamentoso }\end{array}$ \\
\hline 12 & EXPERIMENTAL DE TOXICOLOGIA & Intoxicación por órganofosforados \\
\hline
\end{tabular}

Fuente: Autores 2020 


\section{Marcha experimental de farmacologia y estadistica}

\section{Cálculo de Dosis y Diluciones}

Referencia Teórica:

"La farmacología puede definirse en un sentido más amplio como el estudio de los medicamentos que incluye su historia, origen, propiedades físicas y químicas, presentación, efectos bioquímicos y fisiológicos, mecanismos de acción, absorción, distribución, biotransformación, eliminación y usos con fines terapéuticos." (Chéry, 2010, pág. 3)

Por eso, el conocimiento físico químico básico (peso, volumen) es condición básica necesaria para la determinación de dosis farmacológicas.

\section{Medidas de Peso:}

En Farmacología, se tomará como unidad al gramo (g), que es la milésima parte del Kilogramo. Las medidas de peso se pueden escribir en forma decimal, exponencial, números enteros o fraccionados, por esta razón diremos que en general adquieren diferentes expresiones matemáticas y todas son equivalentes.

$$
\begin{gathered}
0,1 \mathrm{~g}=100 \mathrm{mg}=10 \mathrm{cg}=0,10 \mathrm{~g}=0,001 \\
\mathrm{Kg}=1 \times 10-4 \mathrm{Kg} .
\end{gathered}
$$

Si nos dicen que escribamos $1 / 4$ de miligramo sería: 0,00025 g o 0,25 mg

Calculo de Dosis Total: Al determinar la dosis total necesaria en un paciente, es preciso conocer previamente la dosis/Kg de peso corporal, a la que llamaremos dosis standard y seguidamente aplicamos una regla de tres simple. Por ejemplo: Se desea conocer la dosis total de ampicilina que debe administrarse a un adulto que pesa 87 $\mathrm{kg}$. Si la dosis standard es de $100 \mathrm{mg} / \mathrm{Kg}$. Cuánto deberá administrarse? $\begin{gathered}1 \mathrm{Kg}---100 \mathrm{mg} \\ 87 \mathrm{Kg}---x\end{gathered} \quad x=\frac{87 \mathrm{Kg} \times 100 \mathrm{mg}}{1 \mathrm{~kg}}$

Simplificando magnitudes, tenemos que $X=$ $8700 \mathrm{ng}=8,7 \mathrm{~g}$

Cuando se trata de posología infantil y no se dispone de báscula, o se desconoce el peso del niño, podemos deducir artificialmente en una relación aproximada de dosis pediátrica, "DOSIS RELATIVA". Hay que señalar la sensibilidad o tolerancia de los niños a medicamentos utilizados en adultos.

\section{Medidas de Volumen:}

Farmacología se ha adoptado como unidad el Mililitro ( $\mathrm{ml})$, que es la milésima parte del litro que también puede ser representado como centímetro cúbico (cc). $\quad 1 \mathrm{ml}=1 \mathrm{cc}$

\section{Soluciones y Titulación de Diluciones}

Como concepto general, una solución es la combinación de un disolvente con un soluto. En la mayoría de los casos, el disolvente líquido es agua destilada y el soluto o sustancia disuelta, es una sal o también, otro líquido.

Las soluciones pueden ser: Isotónicas, Hipertónicas o Hipotónicas, de acuerdo a la concentración del soluto en el solvente.

El título de una solución representa la cantidad de una sustancia contenida en un volumen de $1 \mathrm{cc}(\mathrm{ml}), 10 \mathrm{cc}(1 \mathrm{dl})$, o $1000 \mathrm{cc}$ (1 litro). Ejemplo:

Una solución titulada al 5\%, significa 5 gramos disueltos en $100 \mathrm{ml}$ de solución (SOLUCION PORCENTUAL)

Desarrollando tenemos que 0,5 $\mathrm{g}=500 \mathrm{mg}$ contenidos en $100 \mathrm{cc}$. 


\section{Problemas:}

1. Se desea conocer el equivalente $\mathrm{mg} / \mathrm{ml}$ de una solución titulada al $3 \%$ en un volumen de $1 \mathrm{ml}$.

$$
\mathrm{mg} / \mathrm{ml}=3 \times 10 \times 1 \mathrm{ml}=30 \mathrm{mg} / \mathrm{ml}
$$

2. Si la solución de dextrosa en agua viene al 0,45 \%; cuántos mcg existen en $0,5 \mathrm{ml}$ ?

$$
\begin{gathered}
0,45 \mathrm{~g} \\
\mathrm{x}
\end{gathered}
$$

\section{Diluciones:}

En muchos problemas farmacológicos, debido a que algunas soluciones son muy concentradas, o porque el volumen a administrarse es muy pequeño, es preciso añadir un determinado volumen de solvente (volumen adicional) a un milímetro de solución original, con el fin de obtener la solución necesaria, a base de la cual se extrae la dosis calculada en $\mathrm{mg} / \mathrm{ml}$. Ejemplo: Se dispone una solución al 10\% ¿Cómo se procede para convertirla en una solución al 1\%?

En el problema llamaremos:

- $\quad$ So = Solución original.

- $\mathrm{Sn}=$ Título de Solución necesaria

- $\quad$ V1 = Volumen de $1 \mathrm{ml}$ de solución original

- $\quad \mathrm{V} 2$ = Volumen en $\mathrm{ml}$ que es necesario añadir a $\mathrm{V} 1$.

- Para obtener el volumen total:

- $\mathrm{So}=10 \%$

- $\mathrm{Sn}=1 \%$

\section{Dosificación por Gotas:}

Las soluciones en general se dosifican por venoclisis a diversa velocidad de gotas/minutos. En otros casos se emplean equipos cuentagotas, cuando es de menor volumen.

\section{En venoclisis:}

1 ml será igual a 20 gotas.

$$
\begin{aligned}
& x=0,00225 \mathrm{~g} \\
& R=2250 \mathrm{mcg} \text { hay en } 0,5 \mathrm{ml} .
\end{aligned}
$$

1 gota = 3 microgotas (equipo volutrol)

$1 \mathrm{ml} /$ hora $=$ (bomba de infusión)

Objetivo: Reconocer medidas de peso, volumen, soluciones, diluciones, medidas caseras y reducciones entre unidades.

Estrategia: Test diagnóstico. Conferencia-Discusión. Evaluación y Protocolo Experimental.

Resultados: Detectar y realizar reducciones entre medidas de peso, volumen, diluciones, soluciones, medidas caseras, etc.

Conclusiones: Reconocer medidas, diluciones, soluciones y realizar correctamente las reducciones.

\section{Marcha experimental sistema nervioso periferico}

\section{Anestésicos Locales.}

\section{Referencia Teórica:}

"Los anestésicos locales se unen de manera reversible a un receptor específico dentro del poro de los conductos de $\mathrm{Na}+$ en los nervios y bloquean los movimientos iónicos a través de esa abertura. Cuando se aplican al tejido nervioso en concentraciones apropiadas, los anestésicos locales actúan en cualquier parte de tal sistema o en cualquier tipo de fibra nerviosa, y bloquean de modo reversible los potenciales de acción en que se basa la conducción nerviosa. (Hilal-Dandan, Randa ; Brunton, Laurence, 2013). 
Su mecanismo de acción "es el bloqueo de los canales de sodio dependientes de voltaje. El ion sodio tiene una función fundamental en la generación del potencial de acción." (Chéry, 2010)

Objetivo: Demostrar como el anestésico local inhiben la propagación del impulso nervioso sobre la musculatura esquelética (nervio peróneo lateral) al bloquear los neurorreceptores.

Estrategia: Test diagnóstico. Conferencia-Discusión. Evaluación y Protocolo Experimental.

Reactivo Biológico: Rata albina.

Reactivos Farmacológicos: Lidocaina 1\% con epinefrina. Xilocaina 1\% Fco 50ml, Éter , Uretano

\section{Materiales e instrumental:}

Electrodos bipolares de platino

Carrete de Ruthenford (estimulador eléctri$\mathrm{co})$

Instrumental de disección quirúrgica

Tabla de fijación del animal

Algodón - Jeringuillas

Balanza

Seda quirúrgica

Estilete

Procedimiento: Se anestesia al animal, luego Aplicar éter por vía inhalatoria Uretano 10\% 1 sol 1mg por vía IP.

Se diseca el nervio peróneo lateral y la masa muscular adyacente con seda trenzada localizar un segmento del nervio.

a) Testigo, estimulación directa del nervio hasta detectar el umbral de excitabilidad y su respuesta.

b) Problema, se agregan varias gotas de lidocaina en algodón, se espera 5 min. y se estimula eléctricamente el nervio con el vol- taje que anteriormente producía respuesta.

\section{Resultados:}

a) Testigo, umbral de excitabilidad por la corriente eléctrica.

b) Respuesta flexión busca de la extremidad posterior de la rata.

c) Problema aplicando el voltaje anterior de excitabilidad.

Respuesta no hay umbral de excitabilidad ni flexión brusca.

Nota: - Puede ser el lado derecho testigo y el lado izquierdo problema .

Con un solo nervio peróneo lateral $1^{\circ}$ testigo Luego $2^{\circ}$ problema.

Conclusiones: El anestésico local (lidocaína $1 \%$ ) bloquea la conducción nerviosa produciendo insensibilidad y no respuesta motora.

\section{Marcha experimental del sistema nervio- so central}

Anestésicos Generales.

Referencia Teórica:

"Los anestésicos generales producen dos efectos fisiológicos importantes a nivel celular:

Los anestésicos por inhalación hiperpolarizan las neuronas, efecto importante en aquellas que actúan como"marcapasos" y en circuitos que generan pautas o características.

Los anestésicos por inhalación y los que se aplican por vía IV ejercen efectos sustanciales en la transmisión sináptica y otros de menor cuantía en la generación o propagación de potenciales de acción." (Hilal-Dandan, Randa ; Brunton, Laurence, 2013, pág. 327)

Farmacocinética: Se absorben por todas las vías pero como se realiza por vía intravenosa se descarta la absorción. Los órga- 
nos que reciben la mayor cantidad de anestésico son aquellos que son más irrigados como cerebro, corazón, hígado y riñón lo que explica la rapidez de la anestesia. Desde la sangre pasa a las vísceras y tejidos en gran cantidad cayendo la concentración sanguínea. Se metaboliza el anestésico y luego es excretado por la orina.

- Objetivos: Observar los distintos períodos de la anestesia etérea.

- Estrategia: Test diagnóstico. Conferencia-Discusión. Evaluación y Protocolo Experimental.

- Reactivo Biológico: Rata albina.

- Reactivo Farmacológico: Éter.

\section{Materiales e Instrumental:}

- Campana de vidrio.

- Algodón.

- Jeringuilla.

\section{Procedimiento:}

Se introduce el reactivo biológico bajo una campana de vidrio en cuyo interior se encuentra algodón empapado de éter.

Previo a iniciar el experimento se observa reactividad, respuesta a estímulos y frecuencia cardiaca

Una vez, bajo la campana se aprecian las diferentes etapas por las que pasa el animal, que van desde el estado de incoordinación hasta la pérdida del conocimiento con dificultad respiratoria severa y alteraciones en la frecuencia cardiaca.

Se introduce el reactivo biológico y durante minutos y se observan los planos de la anestesia. Al finalizar el animal está inmóvil, arrefléxico y con pupilas dilatadas.

Resultados: Se aprecian los diferentes planos de la anestesia general.

Conclusiones: Se nota que los anestésicos generales son depresores no selectivos del
SNC.

\section{Marcha experimental del sistema nervio- so central}

\section{Anticonvulsivantes}

\section{Referencia Teórica:}

La fenitoína o difenilhidantoína fue sintetizada en 1908 por Bilz, pero su actividad anticonvulsivante no fue descubierta hasta 1938. Desde entonces es el fármaco más utilizado para el tratamiento de las convulsiones, estando indicada en las crisis tonicoclónicas generalizadas, crisis parciales, convulsiones focales y neuralgia del trigémino. Aunque químicamente esta relacionada con los barbitúricos, no pertenece a esa clase de fármacos. La fenitoína tiene también propiedades antiarrítmicas tipo IB.

- Objetivos: Comprobar que los anticonvulsivantes previenen las convulsiones tónico-clónicas.

- Estrategia: Test diagnóstico. Conferencia-Discusión. Evaluación y Protocolo Experimental.

- Reactivos Biológicos: Cobayo.

- Reactivos Farmacológico: Difenilhidantona $®$ Epamin ampolla $5 \mathrm{ml} 25 \mathrm{mg}$.

\section{Materiales e instrumental:}

- Carrete de Ruthenford.

- Electrodos oculares.

- Jaula - Jeringuillas.

- Algodón.

- Alcohol.

\section{Procedimiento:}

a) Animal testigo: inducimos convulsiones tónico-clónicas es el cobayo mediante los electrodos oculares conectados al carrete de inducción o de Rutherford. Se determina el voltaje necesario de menor a mayor.

b) Animal problema nos sirve el mismo co-

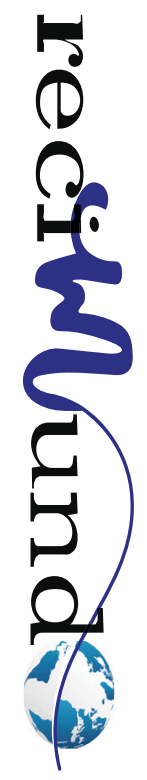


bayo al que administramos Difenilhidantoina IP a Ds $5 \mathrm{mg} / \mathrm{kg}$. Luego de 10 minutos aplicamos el voltaje anterior con los electrodos aculares desde el carrete de inducción.

\section{Resultados:}

a) Animal testigo luego de administrar el voltaje necesario se producen convulsiones tónico-clónicas en el cobayo.

b) Animal problema luego de inyectarse Difenilhidantoina y al administrarse el voltaje anterior y aun elevándolo no se producen convulsiones en dicho cobayo.

Conclusiones: La Difenilhidantoina previene las convulsiones tónico-clónicas.

\section{Marcha experimental sistema nervioso central}

\section{Neurolépticos}

\section{Referencia Teórica:}

Los tranquilizantes más fuertes se usan para los tratamientos de la psicosis y otros transtornos mentales como la esquizofrenia, las manías y el deliriun tremens, éste originado por la abstinencia de bebidas alcohólicas. Entre los más usados se encuentran la reserpina, el haloperidol, la butirofenona, la tioxantina, las fenotiacinas y sus derivados. Esos fármacos se conocen como neurolépticos, que provienen de las palabras griegas neuro ="nervio", y lepto ="atar'.

- Objetivo: Demostrar la acción depresora sobre el SNC de los tranquilizantes mayores.

- Estrategia: Test diagnóstico. Conferencia-Discusión. Evaluación y Protocolo Experimental.

- Reactivo Biológico: Rata Albina.

- Reactivo Farmacológico: Levomepromazina $(R)$ sinogan $25 \mathrm{mg} / \mathrm{ml} /$ ampollas.

\section{Materiales o instrumental:}

- Jaula
- Rejilla de alambre - Balanza.

- Jeringuillas - Cuerda.

\section{Procedimiento:}

- Se pesa a la rata. Se calcuola la dosis total.

- Se administra levomepromazina $\mathrm{Ds}=25 \mathrm{mg} / \mathrm{Kg}$ IP y se contabiliza el tiempo y sintomatología de depresión del SNC.

\section{Resultados:}

\begin{tabular}{|l|l|}
\hline \multicolumn{2}{|c|}{ Periodos de depresión } \\
\hline Tiempo & \multicolumn{1}{c|}{ Cuadro Clínico } \\
\hline I & $\begin{array}{l}\text { Ausencia- del reflejo de fuga (la } \\
\text { rata no huye). }\end{array}$ \\
\hline II & $\begin{array}{l}\text { Disbasia o incoordinación moto- } \\
\text { ra, poca respuestas a estímulos, } \\
\text { táctiles, térmicos y dolorosos (la } \\
\text { rata arrastra el tren posterior o } \\
\text { patas traseras). }\end{array}$ \\
\hline II & $\begin{array}{l}\text { Pérdida del reflejo de sustenta- } \\
\text { ción y mayor incoordinación mo- } \\
\text { tora (la rata no se sostiene de la } \\
\text { rejilla invertida y fácilmente cae) }\end{array}$ \\
\hline
\end{tabular}

Conclusiones: Se comprueba que los neurolépticos (levomepromazina) deprime selectivamente el SNC y en sobredosis puede producir muerte por inhibición de los centros vegetativos del bulbo raquídeo, (Periodo $\mathrm{V}$ o muerte de la rata).

\section{Marcha experimental sistema renal}

\section{Diuréticos}

\section{Referencia Teórica:}

Como se acaba de exponer, son ya varias las familias químicas con capacidad de actuar en el segmento diluyente del asa de Henle. Entre todas ellas, las mejor estudiadas y más utilizadas son los sulfamoilbenzoatos, cuyo representante más característico es la furosemida, y los derivados fenoxiacéticos, especialmente el ácido etacrínico.

Sitio y mecanismo de acción: Son diuréticos 
que producen una diuresis copiosa $y$, en general, de corta duración. Su sitio crítico de acción es el segmento diluyente medular y cortical, y concretamente el epitelio de la porción o segmento grueso de la rama ascendente del asa de Henle, razón por la cual frecuentemente son denominados diuréticos del asa.

Objetivos: Determinar el aumento de la diuresis o excreción urinaria por furosemida.

Estrategia: Test diagnóstico. Conferencia-Discusión. Evaluación y Protocolo Experimental.

Reactivos Biológicos: 2 cobayos (de características semejantes y con buena ingestión de agua).

Reactivos Farmacológicos:

Furosemida R. lasix ampolla $2 \mathrm{ml}=20 \mathrm{mg}$ $\mathrm{Ds}=20 \mathrm{mg} / \mathrm{K}$.

Materiales e Instrumental:

- Vaso pirex con medida

- Embudos

- Rejillas - Soportes

- Balanza

- Jeringuillas

\section{Procedimiento:}

- Animal problema se le inyecta furosemida $20 \mathrm{mg} / \mathrm{kg}$ IP y se lo coloca en el embudo rotulado problema.

- Cobayo testigo se le administra agua destilada IP en la misma cantidad y se lo coloca en el embudo respectivo.

- Se contabiliza la diuresis cada 10 minutos.

\section{Conclusiones:}

a. El animal problema se incrementa la cantidad de orina por efecto de la furosemida que actúa como diurético. b. Animal testigo tiene una diuresis normal y menor al cobayo problema.

\section{Marcha experimental sistema cardiovas- cular}

\section{Cardiotónicos}

\section{Referencia Teórica:}

En pacientes con insuficiencia cardiaca, la digoxina aumenta la fuerza contráctil y el volumen minuto, y disminuye la frecuencia cardiaca, la presión y el volumen telediastólicos ventriculares, la presión capilar pulmonar, la tensión parietal y el índice cardiotorácico. Como consecuencia, mejora los signos de congestión pulmonar y de hipoperfusión tisular y aumenta la capacidad funcional evaluada como tolerancia al ejercicio. El aumento de la contractilidad y del volumen minuto aparece tanto en el corazón normal como en el insuficiente, aunque es mucho más marcado en este último y persiste a lo largo del tiempo, lo que indica que no aparece tolerancia a sus efectos.

Objetivo: Acción de los cardiotónicos sobre el corazón "in situ" de rana.

Estrategia: Test diagnóstico. Conferencia-Discusión. Evaluación y Protocolo Experimental.

Reactivo Biológico: Rana.

Reactivo farmacológico: Digoxina.

Materiales e instrumental:

- Tabla operatoria.

- Instrumental de disección quirúrgica.

- Estilete.

- Quimógrafo -Soportes.

- Palanca de Starling.

- Pluma inscriptora.

- Seda quirúrgica. 


\section{Procedimiento:}

- Se descerebra al animal con un estilete en la región cervical.

- Se fija la rana por sus cuatro extremidades y la tabilla operatoria en decúbito dorsal.

- Se diseca la cavidad toracoabdominal se diseca cuidadosamente el corazón cortando el pericardio.

- Se fija el ápex o punta del corazón con una seda quirúrgica y el otro extremo se une a la palanca de Starling que tiene en su extremo una pluma inscritota que marcara la actividad cardiaca en un quimógrafo.

- Actividad normal por automatismo cardiaco propio.

- Se ponen 1-2-4 gotas de Adrenalina dilución de 1(10)-5 y se grafica.

- Se administra 2 - 4 - 8 gotas de Digoxina en dilucion de 2(10)-4 y se grafica.

\section{Conclusiones:}

a) El corazón tiene automatismo propio (trazado normal).

b) La adrenalina incrementa el trabajo del corazón. Es estimulante cardiaco (inotropismo + , cronotropismo + ).

c) Digoxina es cardiotónico ( inotropismo +, cronotropismo -)

\section{Marcha experimental aparato digestivo}

\section{Antihelmínticos.}

\section{Referencia Teórica:}

Los antihelmínticos son fármacos capaces de eliminar los nematodos cestodes o trematodes, parásitos largos que en general se alojan en órganos y tejidos.

Existen dos tipos de sustancias:

a)Vermicidas: que producen la muerte del helminto.
b)Vermífugos: eliminan el helminto del intestino sin producir su muerte.

Dentro del grupo de antihelmínticos tenemos:

a) Benzoimidazoles = albendazol, tialbendazol.

b) Tetrahidropirimidinas $=$ dietilcarbamicina .

C) Cininas $=$ pirvinio.

d) Hidrocarburos halogenados = tetracloroetileno.

Objetivo: Demostrar la acción vermicida y vermífuga en la lombriz de tierra.

Estrategia: Test diagnóstico. Conferencia-Discusión. Evaluación y Protocolo Experimental.

Reactivo biológico: Lombriz de tierra ( 8 10 ).

Reactivo Farmacológicos: Sol salina fisiológica Fco $500 \mathrm{ml}$.

Albendazol $\otimes_{\text {Susp }} 400 \mathrm{mg}$ Fco $30 \mathrm{ml}$

Pirerazina ${ }^{\circledR}$ Susp 2\% Fco.

\section{Materiales e instrumental:}

- Cajas de Petrie

- Gotero

- Jeringuillas - Algodón

- Alcohol

\section{Procedimiento:}

a. Testigo, poner sol. salina fisiológica $30 \mathrm{ml}$ en una cajá de Petrie y se agrega 2 lombrices de tierra vivas.

b. Problema, poner en una cajá de Petrie $30 \mathrm{ml}$ de Albendazol e introducir 2 vermes.

c. Problema, en una cajá de Petrie poner $30 \mathrm{ml}$ de Piperazina e introducir 2 lombrices de tierra vivas. Luego de 10 minutos lavar lãs 3 cajas de Petrie con sol salina por 3 veces cada una. 
Resultados: Testigo, se mantienen vivos los vermes de tierra.

b. Problema, las lombrices mueren a pesar de los lavados y eliminar el medicamento (acción vermicida).

c. Problema, las lombrices de tierra inmóviles recobran su vitalidad luego de eliminar el fármaco con varios lavados (acción vermífuga).

\section{Conclusiones:}

a.Testigo, vitalidad normal.

b.Albendazol tiene acción vermicida.

c.Piperazina tiene acción vermífuga pues luego de parálisis flácida el reactivo biológico recobra su vitalidad.

\section{Marcha experimental sistema endocrino}

Ocitócicos y útero inhibidores.

\section{Referencia teórica:}

La ocitocina estimula la producción de potenciales espiga en las células miometriales, aumentando la frecuencia de descarga y el número de potenciales presentes en una descarga, así como su amplitud.

Estimulantes 2-adrenérgicos.- Los fármacos empleados son: ritodrina, terbutalina, salbutamol, fenoterol y hexoprenalina.

Acciones farmacológicas.- En el útero grávido, los estimulantes 2 inhiben la contracción uterina, tanto la espontánea como la provocada por diversos estímulos. Por este motivo, según la dosis, aparecen en la madre taquicardia, reducción de la presión diastólica sin modificación de la sistólica y aumento de la presión diferencial.

Objetivo: Acción de las drogas ocitócicas y útero inhibidores sobre el útero.

Estrategia: Test diagnóstico. Conferencia-Discusión. Evaluación y Protocolo Experimental.

Reactivo biológico: cobayo hembra.
Reactivo farmacológico:

Ocitocina $®$ pitocin ampolla $1 \mathrm{ml}=10 \mathrm{ul}$

Fenoferol $®$ ranote

\section{Materiales e instrumental:}

- Equipo de disección quirúrgica

- Oxigeno

- Seda quirúrgico

- Baño Maria

- Quimógrafo

- Soportes

- Palanca de Starling - Pluma inscriptora

- Algodón

- Alcohol

- Caja de Petrie

- Sol salina fisiológica

- Vástago de vidrio

- Sol. Tyrode.

\section{Procedimiento:}

Se descerebra al animal mediante traumatismo cervical.

Se diseca la cavidad abdominal y el útero del cobayo hembra.

Se extraen los cuerpos uterinos y se los coloca en baño de María brindándole las mismas condiciones que el organismo vivo:

A/Temperatura $37^{\circ} \mathrm{C}$ con baño María y comprobar temperatura. B/Oxigenación constante, mediante un vástago de oxígeno. C/ Medio interno con sol. Tyrode semejante al medio fisiológico de la cavidad abdominal.

El extremo de cuerno uterino va unido con seda quirúrgica al vástago de vidrio que da la oxigenación permanente.

El otro extremo va conectada con una seda quirúrgica a la palanca de Starling que tie-

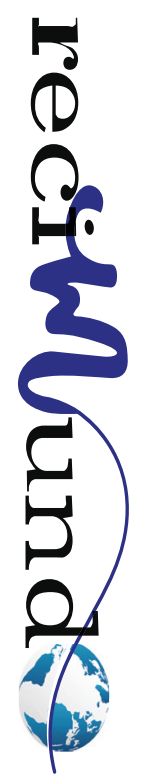


ne en un extremo la pluma inscriptora que marca la actividad muscular en un quimógrafo.

Se grafica cada 2 minutos y se realizan lavados sucesivos

Trazado normal

Ocitocina $0.1 \mathrm{ml}$ se lava

4' ocitocina $0.1 \mathrm{ml}$ se lava 6' fenoterol 0.1 lavado

8' ocitocina + orciprenalina

\section{Resultado y Gráficos:}

a) Trazado normal, el útero tiene contracciones en gestación.

b) Útero estimulación los ocitocina aumenta la altura y el número de espigas o contracciones.

c) Mayor estimulación uterina.

d) Útero inhibición, el fenoterol disminuye el número de contracciones y la fuera de contracción.

e) Estimulación + inhibición, aumenta y cae la curva o contracción uterina.

Conclusiones: En el útero grávido la ocitocina actúa como estimulante de las contracciones uterinas y el fenoterol actúa como útero inhibidor.

\section{Marcha experimental sistema respiratorio}

\section{Histamina y antihistamínicos}

\section{Referencia Teórica:}

La histamina es una amina sintetizada en 1907, que posteriormente fue aislada en los tejidos. Está compuesta por un anillo imidazólico y una cadena lateral etilamino. Se encuentra almacenada principalmente en los mastocitos del tejido conjuntivo y en las células basófilas de la sangre.

Objetivo: Acción de la histamina y antihistamínicos sobre el sistema respiratorio.
Estrategia: Test diagnóstico. Conferencia-Discusión. Evaluación y Protocolo Experimental.

Reactivo biológico: 3 Cobayos.

Reactivos Farmacológicos:

Histamina Amp

Diefenhidramina Amp

Dexametasona Amp

Materiales e instrumental:

- Cámara de nebulización

- Jaula

- Jeringuillas - Algodón

- Alcohol

- Oxigeno

\section{Procedimiento:}

a) Cobayo testigo, se lo coloca en la cámara la de nebulización a una atmósfera de Histamina 1(10)-3, que se nebuliza con una corriente continua de oxígeno y se observa el cuadro clínico asmatiforme que se presenta en el animal. Luego se le administra Salbutamol IP 1 amp.

b) Cobayo problema, se lo introduce en la cámara de nebulización con una atmósfera de Histamina + Dexametasona IP 1 amp.

c) Otro cobayo problema se le administra Histamina en la cámara de nebulización + Difenhidramina IP 1 amp.

\section{Resultados:}

a) Testigo, antagonismo no competitivo pues se produce crisis asmática por Histamina en el cobayo (disnea, tos, tiraje intercostal, aleteo nasal, estridor). El mismo que se elimina con Salbutamol que es broncodilatador.

b) Problema, antagonismo no competitivo pues se produce crisis asmática en el cobayo inducida por histamina la que se elimina 
con corticoides como Dexametasona.

c) Problema, antagonismo competitivo pues el antihistamínico como la Difenhidramina bloquea los receptores por concentración e impide que la Histamina produzca crisis asmática en el cobayo.

\section{Conclusiones:}

- La histamina es un potente broncocotrictor y actúa desencaminado crisis asmática.

- Salbutamol es un broncodilatador que elimina las crisis asmáticas.

- Dexametasona y los corticoides mejoran la crisis asmática al disminuir el edema de la mucosa respiratoria.

- El antihistamínico Loratadina por gradiente de concentración evita que la histamina desencadene crisis asmática.

\section{Marcha experimental aparato digestivo}

Sinergismo y antagonismo medicamentoso.

\section{Referencia teórica:}

La acetilcolina (ACh) es el neurotransmisor específico en las sinapsis del sistema nervioso somático (SNS) y en las sinapsis ganglionares del sistema nervioso autónomo (SNA), así como en los órganos diana de la división parasimpática (ver Ruta 11). La acetilcolina se encuentra también ampliamente distribuida en el encéfalo y es un neurotransmisor clave en la regulación de los niveles de vigilancia y en el funcionamiento de grandes áreas de asociación.

Objetivos: Comprobar el sinergismo y antagonismo en ileon terminal, "in vivo".

Estrategia: Test diagnóstico. Conferencia-Discusión. Evaluación y Protocolo Experimental.

Reactivo biológico: Rata albina.

Reactivos farmacológicos:

Acetilcolina sol $0.002 \%$ dosis $0.5-1-2$ $\mathrm{ml}$

Neostagmine $₫$ Prostigmine sol $0.001 \%$ amp $0.5 \mathrm{mg}=1 \mathrm{ml}$

Atropina sulfato sol $0.1 \%$ dosis $0.1 \mathrm{mg}$ $1 \mathrm{ml}$

Solución tyrode.

\section{Materiales e instrumental:}

- Baño María

- Seda quirúrgica

- Caja de Petrie

- Equipo de disección quirúrgica

- Palanca de Starling - Termómetro

- Pluma inscriptora

- Quimógrafo

- Soportes.

\section{Procedimiento:}

Descerebramos el cobayo mediante técnica de Magnus.

Disecamos la cavidad abdominal y seccionamos $3-6 \mathrm{~cm}$ de ileon Terminal.

Se une el extremo con seda quirúrgica al vástago de vidrio que da oxigenación constante y el otro extremo va unido a una palanca de Starling que tiene es su extremo una pluma inscriptora para marcar el peristaltismo del intestino en el quimógrafo.

El ileon se mantiene "In vivo" puesto que requiere:

1/ Temperatura $37^{\circ} \mathrm{C}$ suministrado por el baño Maria. 2/ Oxigenación continua dada por el vástago de vidrio unida a un tanque de oxígeno. 3/ Sol tyrode o fisiológica semejante a la de la cavidad abdominal de la rata.

Se obtiene un trazado normal por el peristaltismo del intestino.

Sinergismo medicamentoso, se administra

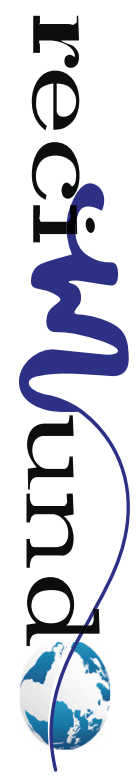


Acetilcolina 1-2-4 mg +

Neostig-

mine $0.5 \mathrm{mg} 1 \mathrm{amp}$.

Antagonismo, Acetilcolina 4mg + Atropina $0.5 \mathrm{mg} 1 \mathrm{amp}$.

Se lava la preparación varias veces luego de cada paso experimental.

\section{Resultados y gráficos:}

a) Trazado normal del peristaltismo.

b) Sinergismo medicamentoso entre la Aceticolina + Neostigmine con mucha elevación de la contracción muscular y de la curva.

c) Antagonismo medicamentoso con caída de la curva y de la contracción por administración de Acetilcolina + Atropina.

\section{Conclusiones:}

a) Sinergismo o aumento de la acción farmacológica por unión de las drogas colinérgicas Aceticolina mas Neostigmine.

b) Antagonismo o disminución de la acción farmacológica entre Acetil colina mas Atropina, esta última es anticolinérgica.

\section{Marcha experimental de toxicologi}

Intoxicación por organofosforados.

\section{Referencia Teórica:}

Derivan de la propiedad fundamental de inhibir la inactivación de la acetilcolina en los sitios donde ésta se libere fisiológicamente, tanto en el SNC como en las terminaciones nerviosas periféricas, somáticas o vegetativas, pudiendo, por lo tanto, producir los siguientes efectos: a)estimulación de los receptores muscarínicos en los órganos efectores vegetativos; b) estimulación, seguida de depresión o parálisis, de todos los ganglios vegetativos y de la musculatura esquelética por activación nicotínica; c) estimulación con depresión posterior ocasional, de receptores colinérgicos centrales. Su acción, por lo tanto, es de carácter colinérgico, pero resulta afectada por algunos factores: a) la capacidad de los compuestos para atravesar membranas: fisostigmina y órgano fosforados no poseen $\mathrm{N}$ cuaternario, por lo que se absorben con facilidad, atraviesan la barrera hematoencefálica y ejercen efectos en el SNC, mientras que la neostigmina y el edrofonio no lo hacen y b) la capacidad de activar directamente receptores nicotínicos, tanto en ganglios como en órganos efectores (p. ej., placa motriz del músculo esquelético).

Objetivo: Demostrar la acción farmacológica (tóxica) del órgano fosforado en el ratón y su tratamiento.

Estrategia: Test diagnóstico. Conferencia-Discusión. Evaluación y Protocolo Experimental.

Reactivo Biológico: 2 lauchas.

Reactivo Farmacológico:

- Paranitrofenildietiltiofosfato (R) Baygon sol $3 \%$ en alcohol etílico

- Atropina (R) atropina sol $1 \%$

- Sol. Salina $0.9 \%$.

Materiales e Instrumental:

- Jaulas

- Jeringuillas

- Rejillas

\section{Procedimento:}

a) Animal testigo, se administra ranitrofenildietiltiofosfato $0.1 \mathrm{ml}$ IP luego se determina el período de latencia hasta la producción de síntomas de toxicidad.

b) Animal problema, dministramos IP paranitrofenildietiltiofosfato $0.1 \mathrm{ml}+$ atropina Ds $25 \mathrm{mg} / \mathrm{kg}$ IP , se espera el período de latencia y la sintomatología.

\section{Resultados:}

a) Animal testigo: Presenta síntomas nicotínicos como temblor, rigidez, fasciculacio- 
nes, hipersensibilidad; síntomas muscarínicos como miosis, salivación, dificultad respiratoria, relajación de esfínteres, llegando a la muerte del animal.

b) Animal problema: No se presentan síntomas y si hay manifestaciones tóxicas se administra más atropina así el animal no muere.

\section{Conclusiones:}

El órganofosforado paranitrofenildietiltiofosfato produce intoxicación con síntomas parasimpáticos o colinérgicos (animal testigo) los que se inhiben o eliminan con atropina (anticolinérgico o parasimpaticolitico). Es decir se produce un antidotismo competitivo en el animal problema.

\section{Conclusiones}

La farmacología experimental o preclínica es el primer paso indispensable para el estudio de los fármacos en humanos. Sin este primer paso como requisito, jamás se podrá llegar al segundo paso experimental (con humanos), mucho menos se podrá distribuir el medicamento, ni lanzarlo al mercado.

Se trata de la salud de la comunidad, del país, del mundo, cuando se está hablando de formación de profesionales en el área farmacológica. Es de suma importancia para garantizar un fármaco de calidad, que minimice efectos colaterales y tenga el efecto esperado en la mayoría de los pacientes, que toda institución que realice estudios de investigación experimental farmacológicos, posea la condición necesaria (infraestructura, materiales, instrumentos, reactivos, reactivos biológicos) para llevar a cabo su misión con éxito.

El Manual De Prácticas Experimentales De Farmacología De La Facultad De Ciencias Médicas De La Universidad De Guayaquil que se planteó, busca estimular la investigación en la Cátedra la Farmacología, en la UG, y sirve como un llamado de atención y de alerta para no dejar caer el propósito y misión de la Facultad. Es prioritario arreglar

\section{Bibliografía}

AVILES, C. (2004). Gestión de Recursos Humanos. Quito: Editorial Universidad Central.

Chéry, P. M. (2010). farmacología básica y clínica. México: McGRAW-HILL INTERAMERICANA EDITORES, S.A. Obtenido de www.rinconmedico.smffy.com

DELGADO, E. (2002). Evaluación de Proyectos. Quito: Asociación de Facultades Ecuatorianas de FiIosofía, Letras y Ciencias de la Educación.

Fernández, P. L., Moreno González, A., Leza Cerro, J. C., Lizasoain Hernández, I., Moro Sánchez, M. Á., \& Portolés Pérez, A. (2009). Velázquez. Farmacología Básica y Clínica (18 ed.). Editorial Médica Panaméricana. Obtenido de https://books.google. co.ve/books/about/Vel\%C3\%A1zquez_Farmaco$\log \% \mathrm{C} 3 \% \mathrm{ADa}$ _B\%C3\%A1 sica_y_Cl\%C3\%ADn. html?id=BeQ6D40wTPQC\&printsec=frontcover\&source $=k p \_r e a d \_b u t t o n \&$ redir_esc $=y \# v=$ onepage\&q\&f=false

FLÓREZ, J. (2016). Pasión por la Farmacología Humana. Generación Elsevier.

FRAGA, C. H. (1997). Modelación del Proceso de formación de Profesionales. Cuba: ISPETP.

Hilal-Dandan, Randa ; Brunton, Laurence. (2013). Goodman \& Gilman. MANUAL DE FARMACOLOGÍA Y TERAPÉUTICA (2da Edicion ed.). California: McGRAW-HILL INTERAMERICANA EDITORES, S. A.

ISCH, E. (2005). Evaluación y los escenarios sociopolíticos a inicios del tercer milenio. quito: Universidad Central del Ecuador.

Litter, M. (1983). Farmacología. Madrid-España: El Ateneo.

LOZADA, B. (2004). Teoría y Diseño Curricular. Quito: Editorial Universidad Central.

Malgor, L. A., \& Valsecia, M. E. (2000). Capítulo 2. Farmacología general: Farmacocinética. Obtenido de Malgor LA, Valsecia ME. Farmacología Médica. 2da ed. (p. 13-32).: http://med.unne.edu.ar/farmaco.html

Meyer, H. H. (1924). La Farmacología experimental como base del tratamiento médico. Tratado para uso de médicos y estudiantes. Editorial Saturnino Callej.

MONTENEGRO, C. C. (2005). Formulación y Evaluación de Proyectos Quito Universidad Central del Ecuador.

MONTENEGRO, M. (2003-2005). Proyecto de Eva-

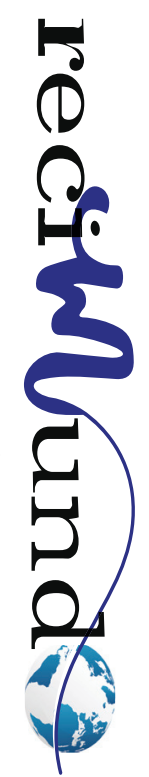


Iuación Intitucional. Quito: Universidad del Norte.

PACHECO, O. (2005). Proyectos Educativos Guayaquil. Guayaquil: Editorial Miinerva.

PORTO, A. (1997). Apuntes para el Estudio de Me- dios en Tecnología Educativa. Habana-Cuba: Editorial ISPETP.

YEPEZ, TERAN, ALEGRÍA. (2004). Planificación Estratégica. Quito: Editorial Universidad Central.

\section{CITAR ESTE ARTICULO:}

Lituma Peñarreta, O., Avalos Moreno, E., Zea Morales, A., \& Castro García, R. (2020). Manual de prácticas de farmacología. RECIMUNDO, 4(1), 249-266. doi:10.26820/recimundo/4.(1).enero.2020.249-266

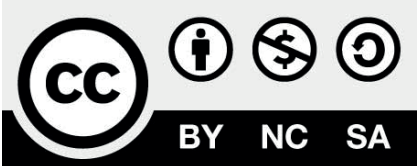

RECONOCIMIENTO-NOCOMERCIAL-COMPARTIRIGUAL CC BY-NC-SA

ESTA LICENCIA PERMITE A OTROS ENTREMEZCLAR, AJUSTARY CONSTRUIR A PARTIR DE SU OBRA CON FINES NO COMERCIALES, SIEMPRE

Y CUANDO LE RECONOZCAN LA AUTORIIA Y SUS NUEVAS CREACIONES ESTÉN BAJO UNA LICENCIA CON LOS MISMOS TÉrMINOS. 